\title{
On the 1-homogeneous non-linear connections
}

Esmaeil Azizpour, Seddighe Darsaraei and Seyed Sajjad Pourmortazavi

\section{ABSTRACT.}

In this paper, we show that for every semispray $S$ on a vector bundle $(R \times T M, \pi, R \times M)$, there are several sequences of semisprays and correspondingly several nonlinear connections associated to it. It is important to derive conditions on $S$, which guarantee that a sequence of nonlinear connections associated to $S$ is constant. We show that the homogeneity condition for $S$ yields the result.

\section{REFERENCES}

[1] Leon, M. De. and Rodrigues, P. R., Dynamical connections and non-autonomous Lagrangian systems, Ann. Fac. Sci. Toulouse Math., (5) 9 (1988), No. 2, 171-181

[2] Miron, R. and Anastasiei, M., The Geometry of Lagrange Spaces: Theory and Applications, Kluwer Academic Publishers, 1994

[3] Vondra, A., Sprays and homogeneous connections on $R \times T M$, Arch. Math. (Brno), 28 (1992), 163-173

Department of Pure Mathematics

UNIVERSITY OF GUILAN, SCIENCES

FACULTY OF MATHEMATICAL

PO BOX 1914, RASHT, IRAN

E-mail address: eazizpoureguilan.ac.ir 